\title{
Management Strategies for POSEIDON's Group 1
}

\author{
Nikolaos P. Polyzos ${ }^{1,2,3 *}$ and Panagiotis Drakopoulos ${ }^{2,4}$ \\ ${ }^{1}$ Department of Reproductive Medicine, Dexeus University Hospital, Barcelona, Spain, ${ }^{2}$ Department of Surgical and Clinical \\ Science, Faculty of Medicine and Pharmacy, Vrije Universiteit Brussel, Brussels, Belgium, ${ }^{3}$ Department of Clinical Medicine, \\ Faculty of Health, Aarhus University, Aarhus, Denmark, ${ }^{4}$ Centre for Reproductive Medicine, Universitair Ziekenhuis Brussel, \\ Vrije Universiteit Brussel, Brussels, Belgium
}

Keywords: poor responders, POSEIDON criteria, suboptimal responders, FSH receptor, LH, FSH polymorphisms, IVF

Poor ovarian responders represent one of the most difficult group of patients in every day clinical fertility practice. Still, a major limitation of the available published research is the striking diversity in the definitions used to define poor ovarian response, which could hamper the validity of the results $(1,2)$.

Despite the recent attempt by the European Society of Human Reproduction and Embryology (ESHRE) to apply a uniform definition for women who respond poorly to ovarian stimulation, the so called "Bologna" criteria (3), it seems that clinicians are still reluctant to use them in clinical studies (4), mainly due to the inability of these criteria to distinguish alterations in oocyte quantity vs. oocyte quality, grouping together women with different biologic characteristics and therefore altered clinical prognosis.

Recently, the POSEIDON group proposed a more detailed stratification of low responders,

OPEN ACCESS

Edited by:

Robert Fischer,

Fertility Center Hamburg

(FCH), Germany

Reviewed by:

Leif Johan Bungum,

Trianglen Fertility Clinic, Denmark

*Correspondence:

Nikolaos P. Polyzos

nikpol@dexeus.com;

n.polyzos@gmail.com

Specialty section:

This article was submitted to

Reproduction,

a section of the journal

Frontiers in Endocrinology

Received: 03 March 2018

Accepted: 18 September 2019

Published: 01 October 2019

Citation:

Polyzos NP and Drakopoulos P (2019)

Management Strategies for

POSEIDON's Group 1.

Front. Endocrinol. 10:679.

doi: 10.3389/fendo.2019.00679 taking into account essential baseline characteristics of infertile women, which could have a significant impact on their reproductive outcome (5). In this context, patient classification is not only based on the number of oocytes retrieved, but also on various other features that may affect treatment success and should be carefully taken into consideration in the era of tailored-approach treatment, such as age and ovarian "sensitivity" to exogenous gonadotropins.

In this regard, four different patients' categories have been identified through the POSEIDON criteria, taking into account patients' age, ovarian reserve markers and response to stimulation in order to define patients' actual prognosis.

POSEIDON Group 1 apparently includes the best prognosis patients, compared to other POSEIDON groups, referring to young infertile women ( $<35$ years old), with adequate ovarian reserve markers $(\mathrm{AFC} \geq 5 ; \mathrm{AMH} \geq 1.2 \mathrm{ng} / \mathrm{ml}$ ), and unexpected poor ( $<3$ oocytes retrieved) or suboptimal (4-9 oocytes retrieved) response following conventional ovarian stimulation (5).

Management of women belonging to the POSEIDON group 1 requires a distinct diagnostic and therapeutic approach in relation to patients' characteristics, which should be specifically tailored to their young age and the adequate ovarian reserve of these women (6).

Age is undeniably the strongest determinant of treatment success in women seeking fertility advice (7). The age-related decline in fertility, owing to a significant decrease in both oocyte quantity (as reflected by lower oocyte yield) and quality (as reflected by higher aneuploidy and spontaneous abortion rates), is directly associated with the very low LBR observed in older women (8). Therefore, although prognosis is very bad in old poor responders, irrespective of the treatment modality used $(9,10)$, substantial benefit could be anticipated in younger women if an adequate number of oocytes is harvested. If we further consider that suboptimal response to stimulation significantly impairs cumulative live birth rates (11-13) and that women with unexpected poor/suboptimal responders may have better prognosis compared to patients with predicted low response (14-16), it could be stated that POSEIDON group 1 patients may represent the most interesting group, on which clinical research should focus in the future. 
Several pathophysiological explanations have been proposed in order to clarify the nature of unexpected poor/suboptimal response. Ovarian sensitivity in relation to gonadotropin treatment has been the dominating theory, with evidence deriving from the investigation of the genetic variations of gonadotropins and their receptors (17). In particular, FSHR polymorphisms (e.g., Ser680Asn and Thr307Ala) have been associated with reduced sensitivity to gonadotropins (18) and may be the most reasonable explanation for the inadequate response following ovarian stimulation (19). This, in addition to the established need for higher gonadotropins in these patients (18), despite their normal ovarian reserve markers (20), suggests that genetic variation in the FSHR is a marker of ovarian sensitivity, irrespective of ovarian reserve.

On the other hand, a common variant of the $\beta$ subunit of luteinizing hormone (LH) (v-LH) has been shown to affect FSH sensitivity and the ovarian response to FSH in normogonadotrophic women. Previous studies demonstrated that patients with this genetic variant of LH may experience an unexpected suboptimal response to stimulation and actually require higher cumulative-dose of gonadotropins $(21,22)$; thus, it may be imperative to consider the potential presence of such a genetic variant among several patients belonging to POSEIDON group 1.

Furthermore, less studied polymorphisms including FSH/LHCGR genes and their combinations may also be relevant, although evidence is sparse $(23,24)$.

Clear treatment guidelines have not been established for POSEIDON group 1 patients; still, this needs to be tailored in accordance to the underlying pathophysiological mechanism responsible for the impaired response to stimulation (6).

Utilization of higher gonadotropin doses of more "potent" recombinant formulations may be the solution in a significant percentage of these women, especially in the ones with polymorphisms identified in the FSHR gene. Taking into account that the Ser680Asn polymorphism of the FSHR gene may negatively influence the ovarian response to $\mathrm{FSH}$ stimulation and women with the genotype Ser/Ser appear to be more resistant to FSH action, a pharmacogenetic study demonstrated that treatment with higher FSH starting dose (225IU) in women homozygous for Ser680 (SS) resulted in similar serum estradiol (E2) levels with women who are homozygous for Asn680 (AA)/heterozygous (AS) treated with lower FSH doses (150IU) and significantly higher E2 levels compared to SS women treated with low 150IU dose (25). Moreover, a recent retrospective study evaluated the effect of FSH dose adjustment in women with a history of suboptimal response (4-9 oocytes retrieved) and demonstrated that an increase in the starting dose of FSH was significantly associated with a higher oocyte yield in the following IVF cycle (26).

On the other hand, administration of $\mathrm{r}-\mathrm{LH}$ supplementation could be another option in these women, especially in cases of genetic variations of LH gene. Given the accumulating evidence from clinical research demonstrating that recombinant $\mathrm{LH}(\mathrm{rLH})$ could potentially increase the number of oocytes retrieved and result in higher pregnancy rates in women with non-pathological ovarian reserve tests and previous unexpected poor (27) or inadequate response (28), the use of rLH in these women is fully justified, and future research is essential to confirm these initial findings.

The utilization of novel promising approaches such a dual stimulation should not be overlooked and may be of benefit for POSEIDON group 1 patients. The rationale of this strategy is that poor prognosis women may undergo both follicular and luteal phase ovarian stimulation in the same menstrual cycle, in an attempt to maximize the number of oocytes retrieved and in turn increase the chance to obtain a genetically normal embryo in a short time interval (29). However, more evidence is needed for the applicability of luteal phase stimulation in poor responders, before implementation in clinical practice.

The synchronization of the follicular cohort through luteal phase estradiol/oral contraceptive pills (OCP) pre-treatment could be an option in young patients with unexpected poor or suboptimal response; albeit evidence extrapolated from studies in poor responders is controversial $(2,30)$.

Finally, adjuvant treatments with growth hormone $(\mathrm{GH})$ or testosterone have been of great interest as an option to improve the outcome in women with a poor ovarian response and certainly merit evaluation in POSEIDON group 1. However, it should be stated that even if previous metaanalyses support the use of these regimens in poor responders $(31,32)$, results need to be interpreted with great caution due to limited evidence and small sample size of the relevant RCTs (33).

In conclusion, young women with normal ovarian reserve markers with a previous unexpected poor or suboptimal response seem to form a distinct group of infertile patients with different clinical prognosis compared to poor responders according to the "Bologna" criteria. Genetic polymorphisms of gonadotropins and their receptors may be a plausible explanation for the poor/suboptimal response following conventional ovarian stimulation; albeit more evidence is needed (NCT03007043, available at: clinicaltrials.gov). The management of these patients may imply the increase in the starting dose of recombinant FSH and/or supplementation with $\mathrm{rLH}$ or even double ovarian stimulation in an attempt to increase the number of oocytes retrieved and therefore the final reproductive outcome. The use of $\mathrm{GH} /$ testosterone and priming protocols including estradiol/OCPs represent other promising options. Nonetheless, further studies are warranted in order to validate these therapeutic approaches.

\section{AUTHOR CONTRIBUTIONS}

NP and PD contributed equally to the design and the writing of the manuscript. 


\section{REFERENCES}

1. Polyzos NP, Devroey P. A systematic review of randomized trials for the treatment of poor ovarian responders: is there any light at the end of the tunnel? Fertil Steril. (2011) 96:1058-61.e7. doi: 10.1016/j.fertnstert.2011.09.048

2. Polyzos NP, Tournaye H. Poor ovarian responders: to metaanalyse or not, that is the question. Hum Reprod. (2014) 29:634-5. doi: 10.1093/humrep/det426

3. Ferraretti AP, La Marca A, Fauser BC, Tarlatzis B, Nargund G, Gianaroli L, et al. ESHRE consensus on the definition of 'poor response' to ovarian stimulation for in vitro fertilization: the Bologna criteria. Hum Reprod. (2011) 26:1616-24. doi: 10.1093/humrep/der092

4. Boza A, Oguz SY, Misirlioglu S, Yakin K, Urman B. Utilization of the Bologna criteria: a promise unfulfilled? A review of published and unpublished/ongoing trials. Fertil Steril. (2018) 109:104-9.e2. doi: 10.1016/j.fertnstert.2017.09.024

5. Poseidon G, Alviggi C, Andersen CY, Buehler K, Conforti A, De Placido $\mathrm{G}$, et al. A new more detailed stratification of low responders to ovarian stimulation: from a poor ovarian response to a low prognosis concept. Fertil Steril. (2016) 105:1452-3. doi: 10.1016/j.fertnstert.2016.02.005

6. Conforti A, Esteves SC, Cimadomo D, Vaiarelli A, Di Rella F, Ubaldi FM, et al. Management of women with an unexpected low ovarian response to gonadotropin. Front Endocrinol. (2019) 10:387. doi: $10.3389 /$ fendo.2019.00387

7. European IVF-monitoring Consortium (EIM), European Society of Human Reproduction and Embryology (ESHRE), Calhaz-Jorge C, De Geyter C, Kupka MS, de Mouzon J, et al. Assisted reproductive technology in Europe, 2013: results generated from European registers by ESHRE. Hum Reprod. (2017) 32:1957-73. doi: 10.1093/humrep/dex264

8. Franasiak JM, Forman EJ, Hong KH, Werner MD, Upham KM, Treff $\mathrm{NR}$, et al. The nature of aneuploidy with increasing age of the female partner: a review of 15,169 consecutive trophectoderm biopsies evaluated with comprehensive chromosomal screening. Fertil Steril. (2014) 101:656-63.e1. doi: 10.1016/j.fertnstert.2013.11.004

9. van Rooij IA, Bancsi LF, Broekmans FJ, Looman CW, Habbema JD, Te Velde ER. Women older than 40 years of age and those with elevated follicle-stimulating hormone levels differ in poor response rate and embryo quality in in vitro fertilization. Fertil Steril. (2003) 79:482-8. doi: 10.1016/S0015-0282(02)04839-2

10. Papathanasiou A, Searle BJ, King NM, Bhattacharya S. Trends in 'poor responder' research: lessons learned from RCTs in assisted conception. Hum Reprod Update. (2016) 22:306-19. doi: 10.1093/humupd/dmw001

11. Polyzos NP, Sunkara SK. Sub-optimal responders following controlled ovarian stimulation: an overlooked group? Hum Reprod. (2015) 30:2005-8. doi: 10.1093/humrep/dev149

12. Drakopoulos P, Blockeel C, Stoop D, Camus M, de Vos M, Tournaye H, et al. Conventional ovarian stimulation and single embryo transfer for IVF/ICSI. How many oocytes do we need to maximize cumulative live birth rates after utilization of all fresh and frozen embryos? Hum Reprod. (2016) 31:370-6. doi: 10.1093/humrep/dev316

13. Polyzos NP, Drakopoulos P, Parra J, Pellicer A, Santos-Ribeiro S, Tournaye $\mathrm{H}$, et al. Cumulative live birth rates according to the number of oocytes retrieved after the first ovarian stimulation for in vitro fertilization/intracytoplasmic sperm injection: a multicenter multinational analysis including approximately 15,000 women. Fertil Steril. (2018) 110:66170 e661. doi: 10.1016/j.fertnstert.2018.04.039

14. Klinkert ER, Broekmans FJ, Looman CW, Te Velde ER. A poor response in the first in vitro fertilization cycle is not necessarily related to a poor prognosis in subsequent cycles. Fertil Steril. (2004) 81:1247-53. doi: $10.1016 /$ j.fertnstert.2003.10.030

15. Klinkert ER, Broekmans FJ, Looman CW, Habbema JD, te Velde ER. Expected poor responders on the basis of an antral follicle count do not benefit from a higher starting dose of gonadotrophins in IVF treatment: a randomized controlled trial. Hum Reprod. (2005) 20:611-5. doi: 10.1093/humrep/ deh663

16. Hendriks DJ, te Velde ER, Looman CW, Bancsi LF, Broekmans FJ. Expected poor ovarian response in predicting cumulative pregnancy rates: a powerful tool. Reprod Biomed Online. (2008) 17:727-36. doi: 10.1016/S1472-6483(10)60323-9

17. La Marca A, Sighinolfi G, Argento C, Grisendi V, Casarini L, Volpe A, et al. Polymorphisms in gonadotropin and gonadotropin receptor genes as markers of ovarian reserve and response in in vitro fertilization. Fertil Steril. (2013) 99:970-8.e1. doi: 10.1016/j.fertnstert.2013.01.086

18. Perez Mayorga M, Gromoll J, Behre HM, Gassner C, Nieschlag E, Simoni M. Ovarian response to follicle-stimulating hormone (FSH) stimulation depends on the FSH receptor genotype. J Clin Endocrinol Metab. (2000) 85:3365-9. doi: 10.1210/jcem.85.9.6789

19. Simoni M, Nieschlag E, Gromoll J. Isoforms and single nucleotide polymorphisms of the FSH receptor gene: implications for human reproduction. Hum Reprod Update. (2002) 8:413-21. doi: 10.1093/humupd/8.5.413

20. Mohiyiddeen L, Newman WG, McBurney H, Mulugeta B, Roberts SA, Nardo LG. Follicle-stimulating hormone receptor gene polymorphisms are not associated with ovarian reserve markers. Fertil Steril. (2012) 97:677-81. doi: 10.1016/j.fertnstert.2011.12.040

21. Alviggi C, Clarizia R, Pettersson K, Mollo A, Humaidan P, Strina I, et al. Suboptimal response to GnRHa long protocol is associated with a common LH polymorphism. Reprod Biomed Online. (2009) 18:9-14. doi: 10.1016/S1472-6483(10)60418-X

22. Alviggi C, Pettersson K, Longobardi S, Andersen CY, Conforti A, De Rosa P, et al. A common polymorphic allele of the LH beta-subunit gene is associated with higher exogenous FSH consumption during controlled ovarian stimulation for assisted reproductive technology. Reprod Biol Endocrinol. (2013) 11:51. doi: 10.1186/1477-7827-11-51

23. La Marca A, Papaleo E, Alviggi C, Ruvolo G, De Placido G, Candiani M, et al. The combination of genetic variants of the FSHB and FSHR genes affects serum FSH in women of reproductive age. Hum Reprod. (2013) 28:1369-74. doi: 10.1093/humrep/det061

24. Riccetti L, De Pascali F, Gilioli L, Santi D, Brigante G, Simoni M, et al. Genetics of gonadotropins and their receptors as markers of ovarian reserve and response in controlled ovarian stimulation. Best Pract Res Clin Obstet Gynaecol. (2017) 44:15-25. doi: 10.1016/j.bpobgyn.2017. 04.002

25. Behre HM, Greb RR, Mempel A, Sonntag B, Kiesel L, Kaltwasser $\mathrm{P}$, et al. Significance of a common single nucleotide polymorphism in exon 10 of the follicle-stimulating hormone (FSH) receptor gene for the ovarian response to FSH: a pharmacogenetic approach to controlled ovarian hyperstimulation. Pharmacogenet Genomics. (2005) 15:451-6. doi: 10.1097/01.fpc.0000167330.92786.5e

26. Drakopoulos P, Santos-Ribeiro S, Bosch E, Garcia-Velasco J, Blockeel C, Romito A, et al. The effect of dose adjustments in a subsequent cycle of women with suboptimal response following conventional ovarian stimulation. Front Endocrinol. (2018) 9:361. doi: 10.3389/fendo.2018. 00361

27. Papaleo E, Vanni VS, Vigano P, La Marca A, Pagliardini L, Vitrano $\mathrm{R}$, et al. Recombinant $\mathrm{LH}$ administration in subsequent cycle after "unexpected" poor response to recombinant FSH monotherapy. Gynecol Endocrinol. (2014) 30:813-6. doi: 10.3109/09513590.2014.9 32342

28. De Placido G, Alviggi C, Perino A, Strina I, Lisi F, Fasolino A, et al. Recombinant human LH supplementation versus recombinant human FSH (rFSH) step-up protocol during controlled ovarian stimulation in normogonadotrophic women with initial inadequate ovarian response to rFSH. A multicentre, prospective, randomized controlled trial. Hum Reprod. (2005) 20:390-6. doi: 10.1093/humrep/deh625

29. Ubaldi FM, Capalbo A, Vaiarelli A, Cimadomo D, Colamaria S, Alviggi C, et al. Follicular versus luteal phase ovarian stimulation during the same menstrual cycle (DuoStim) in a reduced ovarian reserve population results in a similar euploid blastocyst formation rate: new insight in ovarian reserve exploitation. Fertil Steril. (2016) 105:1488-95.e1. doi: 10.1016/j.fertnstert.2016. 03.002

30. Griesinger G, Venetis CA, Tarlatzis B, Kolibianakis EM. To pill or not to pill in GnRH-antagonist cycles: the answer is in the data already! Reprod Biomed Online. (2015) 31:6-8. doi: 10.1016/j.rbmo.2015. 04.001 
31. Duffy JM, Ahmad G, Mohiyiddeen L, Nardo LG, Watson A. Growth hormone for in vitro fertilization. Cochrane Database Syst Rev. (2010) CD000099. doi: 10.1002/14651858.CD000099.pub3

32. Gonzalez-Comadran M, Duran M, Sola I, Fabregues F, Carreras R, Checa MA. Effects of transdermal testosterone in poor responders undergoing IVF: systematic review and meta-analysis. Reprod Biomed Online. (2012) 25:450-9. doi: 10.1016/j.rbmo.2012.07.011

33. Polyzos NP, Davis SR, Drakopoulos P, Humaidan P, De Geyter C, Vega AG, et al. Testosterone for poor ovarian responders: lessons from ovarian physiology. Reprod Sci. (2016) 25:2018. doi: 10.1177/1933719116660849
Conflict of Interest: The authors declare that the research was conducted in the absence of any commercial or financial relationships that could be construed as a potential conflict of interest.

Copyright $\odot 2019$ Polyzos and Drakopoulos. This is an open-access article distributed under the terms of the Creative Commons Attribution License (CC BY). The use, distribution or reproduction in other forums is permitted, provided the original author(s) and the copyright owner(s) are credited and that the original publication in this journal is cited, in accordance with accepted academic practice. No use, distribution or reproduction is permitted which does not comply with these terms. 